\title{
Engineering T cells with hypoxia-inducible chimeric antigen receptor (HiCAR) for selective tumor killing
}

\author{
Qibin $\mathrm{Liao}^{\dagger}$, Huan $\mathrm{He}^{\dagger}$, Yunyu Mao, Xiangqing Ding, Xiaoyan Zhang ${ }^{*}$ and Jianqing $\mathrm{Xu}^{*}$
}

\begin{abstract}
Chimeric antigen receptor-modified T cells (CAR-T cells) have shown good effects in the treatment of hematologic cancers; however, they may cause on-target off-tumor toxicity because of minimal expression of tumor-associated antigens (TAAs) on normal tissues, particularly in the context of treating solid tumors. Hypoxia is a common hallmark of solid tumors because of the Warburg effect. To minimize side effects, we designed a hypoxia-inducible CAR (HiCAR), which is driven by a hypoxia response element (HRE), and consists of a conventional CAR and an oxygen-dependent degradation domain (ODD) that is actively degraded under normoxia but stabilized under hypoxia. HiCAR-T cells showed enhanced cytotoxicity against tumor cells under hypoxia compared to normoxia in vitro and antitumor efficacy comparable to that of conventional CAR-T cells in vivo. Overall, our study demonstrates the potential of the HiCAR for improving the safety of CAR-T cells to promote the clinical application of CAR-T immunotherapy.
\end{abstract}

Keywords: On-target off-tumor, Hypoxia, Hypoxia-inducible CAR, Hypoxia response element

To the Editor,

Chimeric antigen receptor-modified $\mathrm{T}$ cells (CAR- $\mathrm{T}$ cells) have shown strong antitumor activity against hematologic cancers $[1,2]$, which has led to safety concerns regarding on-target off-tumor toxicity, particularly in normal tissues with low expression levels of tumorassociated antigens (TAAs) that are recognized by CAR$\mathrm{T}$ cells $[3,4]$. Recent years have seen the emergence of many strategies to spatiotemporally control CAR-T cell activities through regulating antigen recognition $[5,6]$, but the application of tumor environmental signals (e.g., acidosis and hypoxia) may represent an attractive strategy to control CAR-T cells. A previous study showed that oxygen-sensitive multichain CAR-T cells are responsive to hypoxia [7], while a simple single-chain

\footnotetext{
* Correspondence: zhangxiaoyan@shphc.org.cn; xujianqing@shaphc.org.cn Qibin Liao and Huan He are Co-first authors

Shanghai Public Health Clinical Center \& Institutes of Biomedical Sciences, Fudan University, Shanghai, China
}

hypoxia-inducible CAR-T cells (HiCAR-T cells) could respond to hypoxia within solid tumors is elusive.

We aimed to develop a CAR design that require both antigen recognition and hypoxia sensing to generate optimal $\mathrm{T}$ cell activity, thereby reducing on-target off-tumor toxicity. This design, founded on the oxygen-dependent degradation domain (ODD), might be particularly useful for targeting common antigens shared between normal and neoplastic tissues. We first compared the hypoxiasensitivity of four ODDs, three from hypoxia inducible factor- $1 \alpha$ (HIF- $1 \alpha)$ and one from activating transcription factor-4 (ATF4) [8], using an mCherry-based reporter system (Additional file 1: Figure S1a). Among the four ODDs, the large HIF-1 $\alpha$ ODD conferred the fused reporter gene the lowest baseline expression and the strongest induction under hypoxia condition (Additional file 1: Figure S1b-d). Importantly, the hypoxia-induced mCherry-ODD protein was rapidly downregulated upon return to normoxia (Additional file 1: Figure S1e-g). Consequently, 


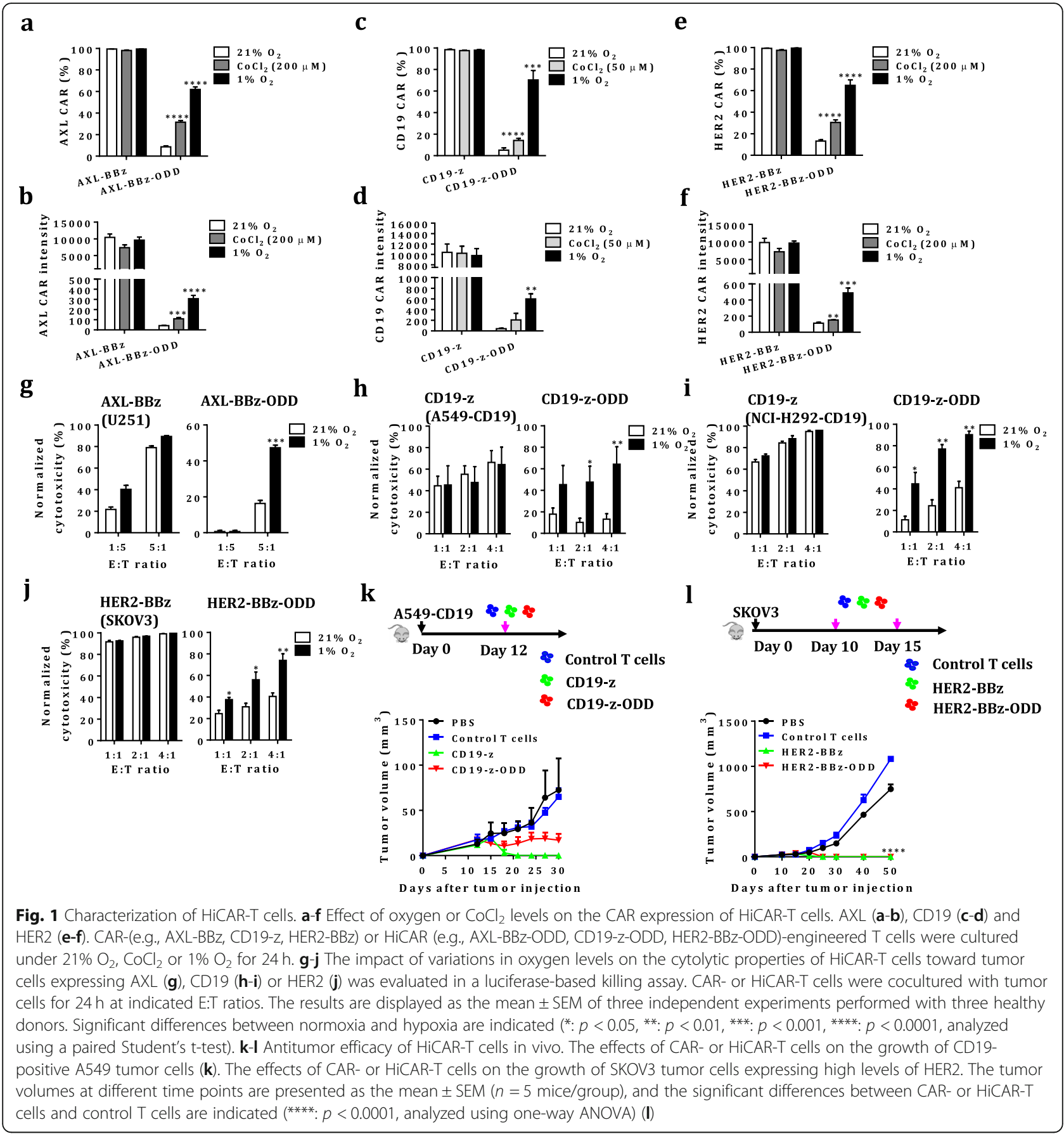

we selected the large HIF-1 $\alpha$ ODD to attach to CARs including CD19 CAR, AXL CAR and HER2 CAR for further testing. Consistent with the reporter studies, the resulting CARs, namely HiCAR, exhibited only minimal basal expression in Jurkat $\mathrm{T}$ cells under normoxia while their surface expression was profoundly enhanced upon hypoxia (Additional file 2: Figure S2).

These data encouraged us to test whether HiCAR could function in primary $\mathrm{T}$ cells. We observed only minimal basal expression under normoxia $(5.1 \%$ and 43 for CD19-HiCAR, $8.6 \%$ and 42 for AXL-HiCAR, and $13.2 \%$ and 113 for HER2-HiCAR, respectively), and hypoxia induced a significant increase in CAR expression, with $70.2 \%$ and 601 for CD19-HiCAR, 61.9\% and 307 for AXL-HiCAR, and $64.9 \%$ and 489 for HER2-HiCAR, respectively (Fig. 1a-f). We further characterized the expression dynamics of CD19-HiCAR protein under hypoxic versus normoxic conditions (Additional file 3: Figure S3a \& S3d). The $t_{1 / 2}$ of the induction of HiCAR by hypoxia, HiCAR-T cells was determined to be $\sim 12 \mathrm{~h}$, with steady 


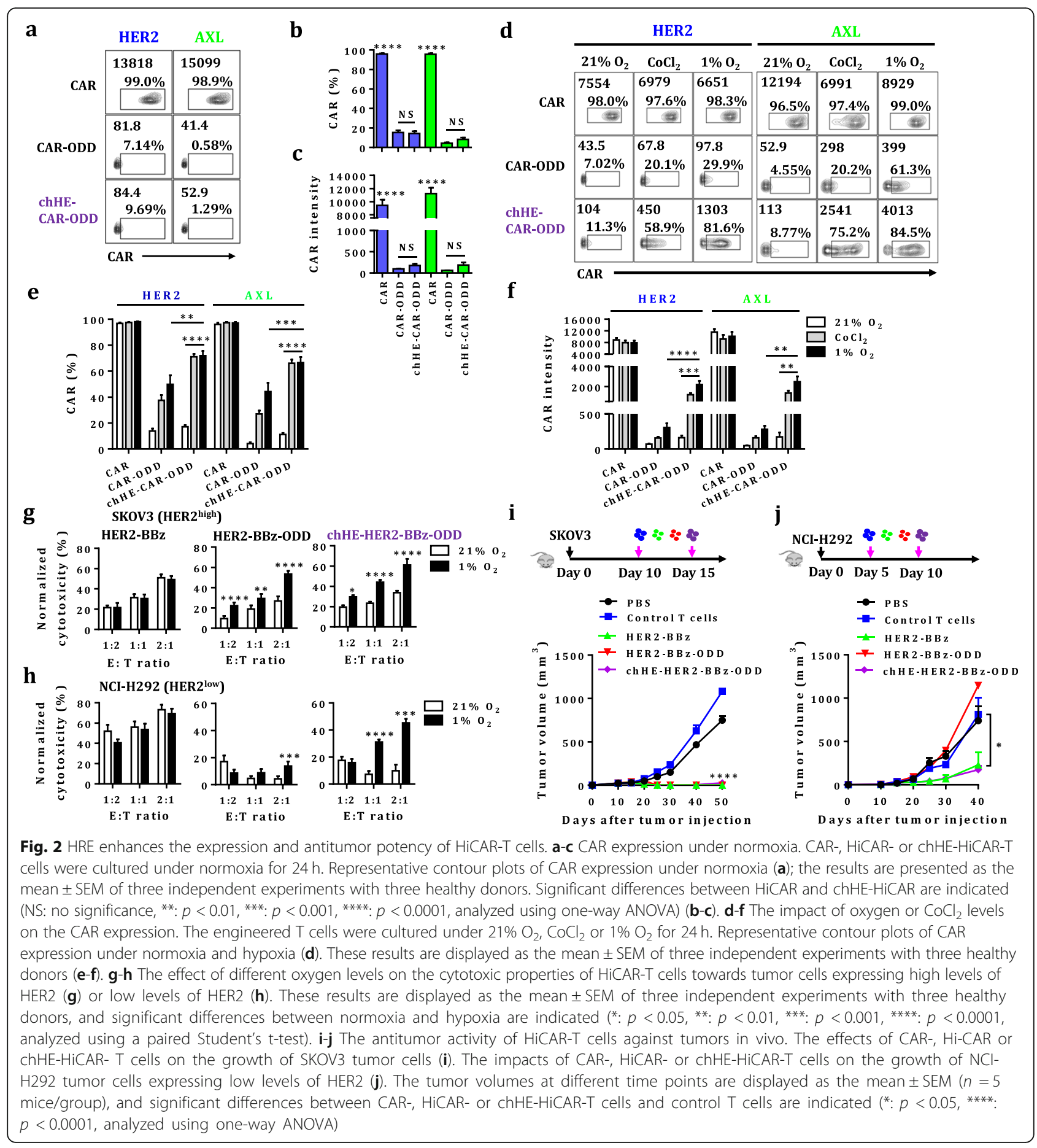

level being reached by $24 \mathrm{~h}$ (Additional file 3: Figure S3b-c) and a reduction of $50 \%$ after the next $24 \mathrm{~h}$. This dynamics is inconsistent with a previous study [7], and different gene delivery methods in our study may account for such discrepancy. As expected, the expression of conventional CAR counterpart was unaffected by the oxygen concentration. Next, we evaluated the controllability of tumor cell-killing activities of HiCAR by oxygen. Notably, for all three examined HiCAR-T cells, only weak cytolytic activities were observed under normoxia, which was dramatically changed upon hypoxia with selective tumor killing being significantly elevated (Fig. 1g-j). We further evaluated the antitumor efficacy of HiCAR-T cells against solid tumors. CD19 HiCAR-T cells showed slightly reduced antitumor activity against CD19-positive tumors compared to their conventional counterparts (Fig. 1k), while HER2 CAR- and 
HiCAR-T cells were comparably effective in the treatment of tumors expressing high levels of HER2 (Fig. 11). It should be noticed that we adopted first and second generation of CAR design respectively for creating CD19 and HER2 HiCAR respectively, indicating ODD fusion as a general approach to achieve oxygendependent activation of CAR-T cells.

As low CAR expression could result in limited antitumor efficacy [9], we intended to improve the hypoxiainduced HiCAR expression by adding a hypoxia response element (HRE) [10] upstream of EF1 $\alpha$ promoter to generate a chimeric HRE-EF1 $\alpha$ promoter (chHE). As expected, chHE-HiCARs were observed to exhibit low basal expression under normoxia (Fig. 2a-c). Importantly, significantly enhanced inductions were observed for chHE-HiCAR in relative to HiCAR, as measured by both percentage of positive cells and mean intensity of fluorescence (Fig. 2d-f). Accordingly, when compared to their HiCAR-T equivalents, chHE-HiCAR-T cells showed augmented cytotoxicity against tumors with low levels of TAAs expression both in vitro (Fig. 2h) and in vivo (Fig. 2j), despite with similar antitumor activities against tumors highly expressing TAAs (Fig. $2 \mathrm{~g}$ and $\mathrm{i}$ ).

We further presented an augmented version of HiCAR, chHE-HiCAR, by placing HiCAR under the control of an HRE-EF1 $\alpha$ promoter, which directs more robust hypoxia-dependent transcriptional upregulation by recruiting hypoxia-inducible factor 1 (HIF-1) transcriptional activator that is accumulated under hypoxia through increased protein stability (Additional file 4: Figure S4). With its clear advantage over conventional CAR- $\mathrm{T}$ cells in the reduction of on-target off-tumor toxicity, HiCAR-T brings new opportunity to immunotherapy for solid tumors.

\section{Supplementary information}

Supplementary information accompanies this paper at https://doi.org/10. 1186/s40364-020-00238-9.

Additional file 1: Figure S1. Identification of the ODD that is responsive to the hypoxic environment. a Schematic representation of various mCherry-ODD reporter constructs. The hypoxia-inducible reporter system contains the reporter mCherry fused with an ODD derived from ATF4 or HIF-1a, including ATF4 ODD (CAG29349.1, 152-186 aa), the Nterminal ODD of HIF-1a (NP_001521.1, 380-491 aa), the C-terminal ODD of HIF-1a (NP_001521.1, 492-603 aa), and a large ODD of HIF-1a (NP_001521.1, 380-603 aa). b mCherry expression in Jurkat T cells transduced with different mCherry-ODD reporter constructs in a normoxic environment. These engineered Jurkat T cells were cultured under normoxia $\left(21 \% \mathrm{O}_{2}\right)$ for $24 \mathrm{~h}$. The expression of mCherry was assessed by flow cytometry. The results are presented as the mean \pm SEM of four independent experiments with technical triplicates, and significant differences in mCherry expression are indicated ( ${ }^{*}: p<0.05,{ }^{* * *}: p<0.001,{ }^{* * *}$. $p<0.0001$, analyzed using one-way ANOVA). c-d These engineered Jurkat T cells were cultured under normoxia $\left(21 \% \mathrm{O}_{2}\right)$ or with cobalt chloride $\left(\mathrm{CoCl}_{2}\right)$ for $24 \mathrm{~h}$. The expression of mCherry was assessed by flow cytometry. The percentages (c) and mean fluorescence intensities (d) of mCherry under chemical hypoxia were normalized to those under normoxia, which are presented as fold-change values. The results are displayed as the mean \pm SEM of four independent experiments with technical triplicates, and significant differences in mCherry expression are indicated (**: $p<0.01,{ }^{* * * *}: p<0.0001$, analyzed using two-way ANOVA). e-g The decay dynamics of mCherry-ODD returned to those under normoxic conditions. Schematic diagram of this decay experiment (e). These engineered Jurkat T cells were cultured under chemical hypoxia for $24 \mathrm{~h}$ and then exposed to normoxia for another $20 \mathrm{~h}$. The percentages (f) and mean fluorescence intensities ( $\mathbf{g}$ ) of mCherry under normoxia were normalized to those at 0 $\mathrm{h}$. These results are presented as the mean \pm SEM of three independent experiments with technical triplicates, and significant differences are indicated $\left(* *: p<0.01,{ }^{* *}: p<0.001,{ }^{* * * *}: p<0.0001\right.$, analyzed using one-way ANOVA).

Additional file 2: Figure S2. Characterization of HiCAR in Jurkat T cells. a-c The effect of cobalt chloride $\left(\mathrm{CoCl}_{2}\right)$ levels on the surface CAR expression of CD19 HiCAR-T cells. Schematic diagram of CD19 CAR and CD19 HiCAR constructs. The CD19-targeting scFv was fused to the CD8 hinge and transmembrane region, followed by the $\mathrm{CD} 3 \zeta$ signaling domain. The ODD was inserted downstream of the CD3Z signaling domain in the conventional CAR to generate the HiCAR construct (a). Jurkat T cells were transduced with either CD19 CAR (CD19-z) or CD19 HiCAR (CD19-z-ODD). The engineered Jurkat T cells were cultured under normoxia $\left(21 \% \mathrm{O}_{2}\right)$ or chemical hypoxia $\left(\mathrm{CoCl}_{2}\right)$ for $24 \mathrm{~h}$. The surface CAR expression was determined by flow cytometry. The results are displayed as the mean \pm SEM of three independent experiments with technical triplicates, and significant differences in CAR expression between normoxia and hypoxia are indicated $\left(* *: p<0.011^{* * *}: p<0.0001\right.$, analyzed using Student's t-test) $(\mathbf{b}-\mathbf{c})$. d-f Effect of cobalt chloride $\left(\mathrm{CoCl}_{2}\right)$ or various oxygen levels on the surface CAR expression of AXL HiCAR-T cells. Schematic diagram of the AXL CAR and AXL HiCAR constructs. The AXL-targeting scFv was fused to the CD8 hinge and transmembrane region, followed by the 4-1BB and $C D 3 \zeta$ signaling domains. The ODD was inserted downstream of the $C D 3 \zeta$ signaling domain in the conventional CAR to generate the HiCAR construct (d). Jurkat T cells were transduced with either AXL CAR (AXL-BBz) or AXL HiCAR (AXL-BBz-ODD). The engineered Jurkat $\mathrm{T}$ cells were cultured under normoxia $\left(21 \% \mathrm{O}_{2}\right)$, chemical hypoxia $\left(\mathrm{CoCl}_{2}\right)$ and physical hypoxia $\left(1 \% \mathrm{O}_{2}\right)$ for $24 \mathrm{~h}$. The surface CAR expression was determined by flow cytometry. The results are presented as the mean \pm SEM of three independent experiments with technical triplicates, and significant differences in CAR expression between normoxia and hypoxia are indicated $\left({ }^{* *}: p<0.01,{ }^{* * *}: p<0.0001\right.$, analyzed using Student's t-test) (ef). $\mathbf{g}$-j Impact of cobalt chloride $\left(\mathrm{CoCl}_{2}\right)$ or various oxygen levels on CAR expression in HER2 HiCAR-T cells. Schematic diagram of the HER2 CAR and HER2 HiCAR constructs as described above $(\mathbf{g})$. Jurkat T cells were transduced with either HER2 CAR (HER2-BBz) or HER2 HiCAR (HER2-BBzODD). The engineered Jurkat T cells were cultured under normoxia (21\% $\left.\mathrm{O}_{2}\right)$, chemical hypoxia $\left(\mathrm{CoCl}_{2}\right)$ and physical hypoxia $\left(1 \% \mathrm{O}_{2}\right)$ for $24 \mathrm{~h}$. Total or surface CAR expression was determined by Western blot analysis (h) or flow cytometry $(\mathbf{i}-\mathbf{j})$. The results are presented as the mean \pm SEM of three independent experiments with technical triplicates, and significant differences in surface CAR expression between normoxia and hypoxia are indicated (**: $p<0.01,{ }^{* * *}: p<0.001,{ }^{* * * *}: p<0.0001$, analyzed using Student's t-test).

Additional file 3: Figure S3. Induction and decay kinetics of HiCAR under hypoxia and normoxia. a Schematic diagram of the induction experiment. b-c CD19 CAR- or CD19 HiCAR-engineered T cells were cultured in normoxic or hypoxic environments for various time points, and the surface expression of CAR was determined using flow cytometry. These results are displayed as the mean \pm SEM of three independent experiments with technical triplicates. d Schematic diagram of the decay experiment. These engineered T cells were cultured under hypoxia for 24 $\mathrm{h}$ and returned to normoxic conditions for $48 \mathrm{~h}$. e Time-course analysis of surface CAR expression decay after returning to the normoxic environment. The percentages and intensities were normalized to $100 \%$ for CAR or HiCAR at the time when the normoxic environment was set up. The results are displayed as the mean \pm SEM of three independent experiments with technical triplicates.

Additional file 4: Figure S4. Schematic diagram of the working principle of HiCAR. In the normoxic environment within normal tissues, 
HiCAR-engineered T cells maintain minimal surface CAR expression using the ubiquitination-proteasome degradation pathway (left panel). Hypoxia is the common hallmark of multiple solid tumors, and increased surface CAR presentation is found on these engineered T cells when they are in the hypoxic environment within solid tumors (middle panel). Insertion of a hypoxia-responsive element (HRE) upstream of the promoter of the lentiviral vector containing the HiCAR construct boosts surface CAR expression to yield enhanced cytolytic potency under hypoxia (right panel).

Additional file 5. Detailed materials and methods.

\section{Abbreviations}

CAR: Chimeric antigen receptor; TAAs: Tumor-associated antigens; HiCAR: Hypoxia-inducible chimeric antigen receptor; HRE: Hypoxia response element; ChHE: Chimeric HRE-EF1a promoter; AXL: AXL receptor tyrosine kinase; HER2: Human epidermal growth factor receptor 2; ATCC: American Type Culture Collection

\section{Acknowledgments}

We thank Lingyan Zhu, Linxia Zhang, and Chenli Qiu for their help with flow cytometry. We thank Lingyan Zhu, Ang Li, and Tianyue Chen for providing advice for performing the experiment. We thank Chen Zhao for help in revising article and language editing. We also thank the Animal Center of Shanghai Public Health Clinical Center and its staff for their help in animal feeding and animal model construction.

\section{Authors' contributions}

JQX and XYZ designed this project and supervised the experiments. QBL and $\mathrm{HH}$ performed the analysis. QBL, $\mathrm{HH}$, and YYM performed the in vitro experiments. QBL and XQD performed the in vivo experiments. All authors read and approved the final manuscript.

\section{Funding}

This work was supported by the National Key Research and Development Program of China (2016YFC1303402), National Megaproject on Key Infectious Diseases (2017ZX10202102, 2017ZX10304402-002-007) and General Program of Shanghai Municipal Health Commission (201740194).

\section{Availability of data and materials}

All supporting data are included in the manuscript and supplemental files. Additional data are available upon reasonable request from the corresponding author.

\section{Ethics approval and consent to participate}

All experimental procedures followed the protocol approved by the institutional ethics committee, and the involved healthy donors provided written informed consent. All animal experiments were performed in accordance with the ethics approval obtained from the Shanghai Public Health Clinical Center Animal Ethics Committee.

\section{Consent for publication}

Not applicable.

\section{Competing interests}

The authors declare that they have no competing interests.

Received: 27 July 2020 Accepted: 30 September 2020

Published online: 30 October 2020

\section{References}

1. Maude SL, Frey N, Shaw PA, Aplenc R, Barrett DM, Bunin NJ, et al. Chimeric antigen receptor $T$ cells for sustained remissions in leukemia. N Engl J Med. 2014:374(16):1507-17.

2. Schuster SJ, Bishop MR, Tam CS, Waller EK, Borchmann P, McGuirk JP, et al. Tisagenlecleucel in adult relapsed or refractory diffuse large B-cell lymphoma. N Engl J Med. 2019;380(1):45-56

3. Lamers $\mathrm{CH}$, Sleiifer S, Vulto AG, Kruit WH, Kliffen M, Debets R, et al. Treatment of metastatic renal cell carcinoma with autologous $\mathrm{T}$ lymphocytes genetically retargeted against carbonic anhydrase IX: first clinical experience. J Clin Oncol. 2006;24(13):e20-2.
4. Morgan RA, Yang JC, Kitano M, Dudley ME, Laurencot CM, Rosenberg SA. Case report of a serious adverse event following the administration of T cells transduced with a chimeric antigen receptor recognizing ERBB2. Mol Ther. 2010;18(4):843-51.

5. Klebanoff CA, Rosenberg SA, Restifo NP. Prospects for gene-engineered T cell immunotherapy for solid cancers. Nat Med. 2016;22(1):26-36.

6. Wang Z, Wu Z, Liu Y, Han W. New development in CAR-T cell therapy. J Hematol Oncol. 2017;10(1):53.

7. Juillerat A, Marechal A, Filhol JM, Valogne $Y$, Valton J, Duclert A, et al. An oxygen sensitive self-decision making engineered CAR T-cell. Sci Rep. 2017; 7:39833.

8. Cho SH, Oh B, Kim HA, Park JH, Lee M. Post-translational regulation of gene expression using the ATF4 oxygen-dependent degradation domain for hypoxia-specific gene therapy. J Drug Target. 2013;21(9):830-6.

9. Walker AJ, Majzner RG, Zhang L, Wanhainen K, Long AH, Nguyen SM, et al. Tumor antigen and receptor densities regulate efficacy of a chimeric antigen receptor targeting anaplastic lymphoma kinase. Mol Ther. 2017; 25(9):2189-201.

10. Kim H, Peng G, Hicks JM, Weiss HL, Van Meir EG, Brenner MK, et al. Engineering human tumor-specific cytotoxic $T$ cells to function in a hypoxic environment. Mol Ther. 2008;16(3):599-606.

\section{Publisher's Note}

Springer Nature remains neutral with regard to jurisdictional claims in published maps and institutional affiliations.

\section{Ready to submit your research? Choose BMC and benefit from:}

- fast, convenient online submission

- thorough peer review by experienced researchers in your field

- rapid publication on acceptance

- support for research data, including large and complex data types

- gold Open Access which fosters wider collaboration and increased citations

- maximum visibility for your research: over $100 \mathrm{M}$ website views per year

At BMC, research is always in progress.

Learn more biomedcentral.com/submissions 